\title{
Rabelais: entre le signal et l'indice
}

\author{
PIERRE GOUMARRE
}

La critique littéraire, qui a toujours été plus ou moins ouverte aux vents nouveaux, est aujourd'hui vivifiée par l'air frais qui souffle en provenance de la sémiologie. L'auteur de Proust et les signes, Gilles Deleuze, a été un des grands pionniers de cette tendance, qui consiste à analyser une oeuvre à travers ses signes. Les quelques constatations et hypothèses qui fondent sa méthode sont simples et raisonnables: "Le mot 'signe' est un des mots les plus fréquents de la Recherche. . Cette oeuvre se présente comme l'exploration des différents mondes de signes. . .Les signes [en] forment à la fois l'unité et la pluralité. . .Le problème de Proust est celui des signes en général."'1

Ces constatations et ces hypothèses peuvent tout aussi bien être faites à propos de l'oeuvre de Rabelais. Tout comme celui qui s'est consacré à la recherche du temps perdu, l'écrivain qui s'est intéressé à la recherche de la dive bouteille a été un grand créateur et un grand explorateur de signes. Du côté de chez Jean comme du côté de chez Swann, dans les controverses de Thaumaste et de Panurge comme dans les causeries de Charlus et de Verdurin, l'univers créé par le génie est un royaume de signes. ${ }^{2}$

Comme la plupart des hommes de son époque, Rabelais s'est beaucoup intéressé aux signes qui permettent de prévoir l'avenir. Tout en étant une parodie des livres de prédiction, la Pantagrueline Prognostication témoigne de l'intérêt de l'auteur pour les présages. Et le Tiers Livre est tout entier consacré à une exploration de l'avenir conjugal de Panurge. Dans ce livre, tous les signes imaginables sont sollicités, y compris ceux que fournissent les rêves. " "En songeant," explique Pantagruel, "l'âme souvent prevoit les choses futures." 4

Mais les signes annonciateurs de l'avenir ne sont nullement les seuls signes de l'oeuvre. Rabelais s'est tout autant intéressé aux signes révélateurs de la réalité présente. Et il est remarquable que, pour lui, tout peut constituer un signe: un geste ou une attitude; un objet ou un produit; un événement social ou un phénomène naturel; un nombre ou un nom, particulièrement un nom propre; une devise, un blason, un emblème, un insigne et une enseigne; un texte, mais aussi une image ou un tableau; une odeur 
aussi bien qu'un son ou une couleur; un sentiment, une impression, une sensation et, bien sûr, un symptôme.

Dans le cadre limité d'un article, il ne saurait être question de se livrer à une exploration systématique de l'univers rabelaisien du signe. Ce n'est pas en quelques pages qu'on peut révéler toute la richesse sémiologique d'une oeuvre, surtout lorsque cette oeuvre est placée sous le signe du gigantisme. Ce que nous nous proposons de faire ici, c'est seulement de nous demander dans quelle mesure les signes de Rabelais relèvent de la sémiologie.

Si en effet l'on en croit plusieurs sémiologues contemporains, la sémiologie n'est pas la science de tous les signes, mais la science des signes qui constituent des moyens de communication. Il est vrai que, dans le langage courant, on appelle souvent signes des faits qui n'ont rien à voir avec la communication. Mais, dans le langage sémiologique, ces faits sont des indices et non des signes. La distinction entre les divers signes, les sémiologues la font surtout en opposant l'indice au signal. Comme l'écrit Mounin, "toute sémiologie correcte repose sur l'opposition catégorique entre les concepts cardinaux d'indice et de signal." Etant un moyen de communication, le signal relève de la sémiologie; n'ayant qu'une valeur d'information, l'indice n'en relève pas.

Définissant l'indice comme "un fait observable qui nous renseigne sur un autre qui ne l'est pas," Mounin précise: "L'interprétation correcte de la signification des indices, c'est toute la science, ce n'est pas la linguistique ni même la sémiologie. Et l'interprétation des indices est une activité profondément différente du décodage des signes. C'est toute la méthode scientifique et l'épistémologie qui l'enseignent"' (p. 194).

C'est certainement Prieto qui a le plus clairement distingué indices et signaux. L'indice, écrit-il, est "un fait immédiatement perceptible qui nous fait connaître quelque chose à propos d'un autre qui ne l'est pas." 6 Quant au signal, c'est "un indice artificiel, c'est-à-dire un fait qui fournit une indication et a été produit expressément pour cela." 7

Dans un livre récent, où elle définit judicieusement le signe comme "quelque chose de perceptible qui rend manifeste autre chose qui, autrement, ne le serait pas," Jeanne Martinet éclaire cette distinction par une réflexion sur la fumée. "Presque tous les auteurs," écrit-elle, "s'accordent pour donner, comme exemple typique de l'indice, la fumée." Et il est vrai qu'en général, la fumée est un indice: "L'émetteur de la fumée est le feu lui-même, et comme le feu ne choisit pas d'émettre ou non de la fumée, on ne saurait lui reconnaître un comportement sémiologique." Mais la fumée "peut être produit[e] artificiellement et utilisé[e] à des fins sémiologiques": dans un conclave, par exemple, "on produit à volonté une fumée noire ou blanche pour signaler l'issue d'un vote." ${ }^{8}$ Dans ce cas, la fumée est un signal.

Exprimant le point de vue du psychologue, Norbert Sillamy distingue l'indice du signal en réfléchissant sur l'exemple des traces des pieds sur le 


\section{8 / Renaissance and Reformation}

sol. "Une empreinte dans la neige," écrit-il, "est l'indice du passage d'un animal." Mais si "je laisse des traces derrière moi pour retrouver mon chemin dans la forêt," j'utilise les traces comme un signal. Cet auteur explique que "l'indice se distingue du signal en ce sens que celui-ci est lié intentionnellement à l'objet ou à la situation." 9

L'oeuvre de Rabelais est très riche de signes, et cette profusion est naturellement source de difficultés. Elle suscite au moins une question: ces signes sont-ils vraiment des signes, c'est-à-dire, dans le langage de beaucoup de sémiologues, des signaux? Ou constituent-ils au contraire des indices? C'est à cette question que nous allons maintenant essayer de répondre.

***

Le signe rabelaisien est souvent un signe au sens sémiologique du terme, et c'est ce que nous allons d'abord montrer. Pour cela, nous choisirons un exemple dans chacun des livres de l'oeuvre.

Dans le Gargantua, les signes apparaissent en particulier sous forme d'anneaux: "Pour ses aneaulx (lesquelz voulut son pere qu'il portast pour renouveller le signe antique de noblesse) il eut. . .une escarbouche grosse comme un oeuf d'austruche." $(I, 39)$ Ce "signe antique" correspond exactement à la définition que Mounin donne du signal: "Un indice produit volontairement par l'émetteur pour manifester une intention au récepteur" (op. cit., p. 14). C'est évidemment Grandgousier qui est ici l'émetteur, et l'entourage de Gargantua qui constitue le récepteur. La volonté de l'émetteur s'exprime naturellement dans le verbe "voulut," et son intention est tout aussi explicite: il s'agit de rappeler à tous que le jeune géant fait partie de la noblesse.

C'est dans le Pantagruel que se déroule la controverse "par signes" où Thaumaste et Panurge sont tour à tour émetteurs et récepteurs. Ces signes sont certes très particuliers, et Chesneau en a dégagé le caractère paradoxal dans une formule lumineuse: ces "gestes-signaux," écrit-il, "présentent un degré plein de signifiant (puisque la gesticulation existe) allié à un degré zéro de signifié (puisque cette gesticulation peut vouloir dire n'importe quoi, c'est-à-dire n'a aucun sens)."'10

Mais, malgré ce paradoxe, ces gestes sont bien des signes de communication. Ce que veulent Thaumaste et Panurge, c'est, selon les termes mêmes de Rabelais, "disputer," "arguer," c'est-à-dire communiquer. S'ils choisissent les gestes de préférence aux mots, c'est qu'ils voient dans les premiers des moyens de communication supérieurs aux seconds. Pour eux, les mots sont, comme le dit Chesneau, des "instruments de communication insuffisants" 11 (Op. cit., p. 36). Le philosophe anglais et le compagnon de Pantagruel échangent des idées et ils se comprennent, c'est du moins ce qu'ils prétendent, même si Rabelais nous avertit de toutes les façons qu'il 
n'y a pas plus à comprendre dans les signes cabalistiques de l'un que dans les gestes obscènes de l'autre.

Les gestes de Nazdecabre, au Tiers Livre, sont tout aussi bien des signes de communication. L'émetteur étant sourd et muet, ils sont même pour lui le seul moyen de communication possible. Il est vrai que les deux récepteurs n'attribuent pas la même signification à son message:

Alors dist Pantagruel: "Si les signes vous faschent, ô quand vous fascheront les choses signifiées!. . .Le mut praetend et denote que serez marié, coqu, battu et desrobbé. - Le mariage (dist Panurge) je concede, je nie le demourant. $(I, 487)$

Mais, tout en étant en désaccord sur le contenu du message, Pantagruel et Panurge sont d'accord pour considérer qu'il y a un message, et que Nazdecabre a bien eu l'intention de communiquer un message. ${ }^{12}$

Dans le Quart Livre, également, on trouve plusieurs signes de communication et c'est par exemple le cas lorsque l'auteur parle de ces juges qui "Ballottans pour le judgment des criminelz prisonniers, usoient de certaines notes scelon la varieté des sentences, par $\Theta$ signifians condemnation à mort; par T , absolution..." (II, 120) En précisant que "icelles, publiquement exposées, houstoient d'esmoy et pensement les parens, amis et aultres," l'auteur montre que ces "notes" étaient bien ce que Prieto appelle des "indices artificiels," c'est-à-dire des indices produits volontairement par les émetteurs (les juges) pour communiquer un message (leurs sentences), et reconnus comme tels - i.e. comme des moyens de communication - par les récepteurs (les parents et les amis des prisonniers).

En d'autres termes, ces notes, que Rabelais appelle des signes, constituent des signaux au sens que la sémiologie moderne donne à ce mot. Et on peut en dire autant de tous les signes que nous avons considérés jusqu'ici: étant des instruments de communication, ils relèvent de cette science dont l'objet même est la communication, et que depuis Saussure on appelle la sémiologie. ${ }^{13}$ Pour employer une expression de Buyssens, ils sont "conventionnels, en ce sens que les individus sont d'accord pour les utiliser tels qu'ils sont." ${ }^{4}$ Emis comme des moyens de communication, ils sont reçus comme tels.

$* * *$

Mais, à côté de ces signes conventionnels, dans lesquels Buyssens voit des "signes sémiotiques," il y a ce que cet auteur appelle des "signes naturels." Comme les premiers, ces derniers peuvent présenter un intérêt considérable, puisqu'ils peuvent fournir toutes sortes d'indications. Mais ils n'ont rien à voir avec la communication, et par là se situent en dehors du champ de la sémiologie. On peut les appeler des signes, on peut dire qu'ils ont une signification, mais c'est là, selon l'expression de Mounin, 
"une polysémie désastreuse" (Op. cit., p. 194). Car, pour beaucoup de sémiologues du moins, ce ne sont pas des signes, mais des indices. Quel emploi Rabelais fait-il des indices? Comment les conçoit-il? Comment les nomme-t-il? Telles sont les questions auxquelles il convient maintenant de répondre.

Rabelais n'aime pas le mot indice, mais il ne l'ignore pas. La preuve en est ce passage du Tiers Livre: "Si mouvement propre est indice certain de chose animée, comme escript Aristocles. .." (I, 540) Le mouvement propre est proprement un indice en effet: le mouvement peut fournir une indication, mais ne permet évidemment pas de communication. Il se trouve du reste que cet indice est vide de contenu puisque, inséré dans une tautologie, il fournit une indication qui est évidente. Indice à degré zéro de signifié, comme dirait Chesneau, et qui ne provient même pas de Rabelais, mais d'Aristote. Indice en tout cas, et non pas signal.

Il est des indices qui fournissent des informations plus nombreuses, et surtout plus utiles, à savoir les symptômes. Les sémiologues n'ignorent pas ce terme, et certains en font même un simple synonyme d'indice. C'est ainsi que Mounin établit une distinction rigoureuse entre "conventions ou signes" et "indices ou symptômes" (Op. cit., p. 69). Ces faits, qu'on peut appeler indices ou symptômes, il les définit comme "des renseignements que le locuteur donne sur lui-même, sans aucune intention de les communiquer" (p. 68).

Le docteur Rabelais s'intéresse naturellement aux symptomes, mais il les considère au sens courant du terme, c'est-à-dire comme des renseignements donnés non par le locuteur, mais par le malade. C'est en ce sens qu'il voit "un des symptômes et accidens de paour" dans le fait que "se ouvre le guischet du serrail [où] est. . .la matière fecale retenue" (II, 245). Ce symptôme est un indice qui relève de la scatologie, un indice néanmoins, qui ne relève donc pas de la sémiologie.

Le plus souvent, toutefois, Rabelais appelle les symptômes non des indices - ni tout simplement des symptômes -, mais des signes. C'est ainsi que le seigneur de Baisecul parle de "physiciens" qui, d'après lui, avaient vu dans "l'urine" de quelqu'un un "signe evident" que. . (I, 276) Il est vrai que cette expression sort de la bouche de Baisecul, et qu'il sort de cette bouche beaucoup de sottises. Mais on retrouve ailleurs le même emploi du mot signe, et dans un contexte tout à fait sérieux: "Le prudent médecin, voyant par les signes prognostiez son malade entrer en decours de mort, par quelques jours davant avertist les femme, enfans. .." (II,119)

Il est évident que Rabelais ne fait pas beaucoup d'efforts pour distinguer indices et signes. Confusion très grave d'après Mounin: "Si nous devions admettre une terminologie qui confond symptomes, indices et signes, nous devrions dire (jamais plus un linguiste ne l'acceptera) que la maladie communique avec le médecin" (p. 68). 
Mais Mounin jugerait-il cette confusion aussi sévèrement s'il songeait aux enseignements de la médecine psychosomatique? Dans l'esprit de cette discipline, il est permis de penser que les symptômes sont d'une certaine façon, pour le malade, un moyen de révéler ses troubles, et n'importe quel linguiste peut accepter ce point de vue. Rabelais l'accepte en tout cas, et cela ne prouve ni qu'il est mauvais médecin, ni qu'il est mauvais sémiologue. Si cela prouve quelque chose, c'est plutôt que, dans ce domaine comme dans beaucoup d'autres, il est en avance sur son temps.

Ce qui est certain, c'est que Rabelais se refuse à établir une frontière précise entre le signe et l'indice. Ce refus, nous allons le voir, est encore plus manifeste dans deux expressions qu'il employe dans le Tiers Livre.

Voici ce que dit Panurge: "Vray est qu'en moy je recongnais quelque signe indicatif de vieillesse, je diz verde vieillesse. . C'est que je trouve le vin meilleur. .que [d'habitude]." (I, 520) Considérant que le vin n'est pas un moyen de communication, un sémiologue verrait naturellement, dans le plaisir de boire du vin, un indice. Pour Rabelais, ce plaisir est-il un signe ou un indice? Ni l'un ni l'autre, ou l'un et l'autre: c'est un "signe indicatif."

Un signe donnant une indication: se rapportant au vin, cette expression peut choquer plus d'un sémiologue. Mais il faut voir que, dans le contexte de l'oeuvre rabelaisienne, elle est tout à fait logique. Un signe? Oui, un signe, car pour l'auteur, si du moins l'on en croit la Pontife Bacbuc, le vin a un pouvoir de communication: "Pouvoir il a d'emplir l'ame de toute verité, tout savoir et philosophie." (II, 454) Une indication? Oui, une indication, et qui a l'avantage d'être très claire, si du moins l'on se fie aux précisions que Panurge apporte sur sa "verde vieillesse": "Tu me reproches mon poil grisonnant et ne consydere poinct comment il est de la nature des pourreaux, es quelz nous voyons la teste blanche et la queue verde, droicte et viguoureuse."

L'autre expression, nous la devons à Pantagruel: “C'est pourquoy Heraclitus disoit rien par songe ne nous estre exposé, rien aussi ne nous estre celé: seulement nous estre donnée signification et indice des choses advenir." (I, 454) Alors qu'un sémiologue trouverait dans un rêve des indices plutôt que des signes, Rabelais parle, lui, de "signification et indice," et le mot signification, suggérant qu'il y a signe, peut paraître de trop.

Mais n'est-on pas en droit de penser, trente ans après la mort de Freud, que les rêves ont une signification, et qu'ils constituent même, en quelque sorte, un moyen de communication? Tel est bien en tout cas le point de vue de Rabelais: réfléchissant sur la nature et l'origine des songes, il explique en effet que l'âme, recevant de Dieu connaissance des choses passées et futures, nous les "raporte" "comme la lune, recevant du soleil sa lumière, nous la communique." (I, 453) L'hésitation de l'auteur, à propos des rêves, entre l'indice et le signe, n'a rien de choquant: qu'on accepte ou non les 
théories freudiennes, on sait aujourd'hui que le rêve est un phénomène complexe, sur la nature duquel il est permis d'hésiter.

Les quelques passages que nous avons cités montrent que Rabelais n'établit pas une distinction nette entre le signe et l'indice. Alors qu'il n'employe le mot indice qu'à deux reprises, il employe à chaque instant le mot signe, mais ce mot apparaît souvent dans des cas où un sémiologue parlerait d'indice. Rabelais parle de signe dans des cas où un fait de communication est évident, mais aussi dans des cas où ce fait est discutable, et même dans des cas où ce fait serait difficile à trouver.

Avant toutefois de lui reprocher cette confusion, il faut voir qu'on ne la trouve pas que chez lui. On la trouve par exemple chez un linguiste aussi distingué que Benveniste, comme en témoigne la définition qu'il donne du mot signal: "C'est un fait physique relié à un autre fait physique par un rapport naturel ou conventionnel."15 Au contraire de Buyssens et de beaucoup d'autres sémiologues, Benveniste refuse, on le voit, de distinguer les signes naturels et les signes conventionnels ou sémiotiques.

Et les exemples de signaux cités par lui montrent toute la portée de ce refus: "éclair annonçant l'orage, cloche annonçant le repas, cri annonçant le danger." Pour Benveniste, l'éclair est un signal au même titre que la cloche et que le cri: c'est dire que, selon lui, il n'y a pas de différence essentielle entre l'indice et le signal. Rabelais, de même, énumère une liste de faits où le naturel et le conventionnel font bon ménage: "grand troublemens, estonnemens. . . fortunal et tempestes, avec que lamentation des peuples, mutations des religions, transpors des Royaulmes, et eversions des Républicques." (I, 117) Et il précise que ce sont là des "signes" par lesquels "les cieulx disent tacitement. ..." Comme quoi il est décidément impossible, dans son oeuvre, d'apercevoir une distinction entre l'indice et le signal, entre la simple information et la communication.

Rabelais a-t-il eu raison de refuser ainsi de faire cette distinction? L'état actuel de la sémiologie ne nous permet pas de répondre à cette question puisqu'il est des sémiologues - comme Prieto - qui font cette distinction, et d'autres - comme Benveniste - qui ne la font pas. De toute façon, à propos de Rabelais, on aurait mauvaise grâce à invoquer l'argument d'autorité. Seul, évidemment, un examen de la réalité pourrait nous fournir la réponse. La distinction rigoureuse entre l'indice et le signal est-elle justifiée par les faits? Telle est la question qu'il faudrait se poser. A cette question nous n'avons apporté, dans notre ètude, qu'un début de réponse. Nous avons trouvé ce début dans l'oeuvre de Rabelais, et cela suggère que toute la réponse s'y trouve aussi. Nous en sommes du reste convaincu: précieuse pour toutes les sciences humaines, l'oeuvre de Rabelais est aussi, naturellement, précieuse pour la sémiologie. 


\section{Renaissance et Réforme / 23}

Notes

1 Gilles Deleuze, Proust et les signes (P.U.F., 1971).

2 A propos de Rabelais et la sémiologie, il faut lire:

- Jean Paris, Rabelais au futur (Paris: Seuil, 1970).

- Jean-Claude Raillon, “Le signe renaissant," Marche Romane, 1971, n 4, pp. 21-35.

- Floyd Gray, Rabelais et l'Ecriture (Paris: Nizet, 1974).

- François Rigolot, "Cratylisme et Pantagruclisme: Rabelais et le statut du signe," Etudes Rabelaisiennes (Genève: Droz, 1976), pp. 115-132.

3 Il est sur cette question un article remarquable de M.L. Goodrich: "The Dream of Panurge," Etudes Rabelaisiennes (Genève: Droz, 1967), pp. 93-103.

4 Rabelais, Oeuvres Complètes (Garnier, 1962), Tome I, p. 452. Toutes les citations utilisées dans cet article proviennent de l'édition Garnier.

5 Georges Mounin, Introduction à la sémiologie (Editions de Minuit, 1970), p. 13.

6 Définition prise dans l'article intitulé "Sémiologie" (p. 95) et citée par Mounin, op. cit., p. 13.

7 Luis J. Prieto, Messages et signaux (P.U.F., 1966), p. 19, n.1.

8 Jeanne Martinet, La Sémiologie (Paris: Seghers, 1973), pp. 54-58.

9 Norbert Sillamy, Dictionnaire de la Psychologie (Paris: Larousse, 1965), p. 150. Cet auteur distingue ailleurs le signal du signe: "A la différence du signal, qui s'adresse au réflexe et à l'inconscient, le signe fait appel à l'intelligence" (p. 277). Mais cette distinction n'a pas cours en sćmiologie. Certains sémiologues emploient le terme "signal" et d'autres le terme "signe," mais les uns et les autres veulent dire la même chose.

10 Albert Chesneau, "Un point de sémiologie pantagruélique: Problèmes et valeur du langage par signes selon Rabelais," Bulletin des jeunes Romanistes (No. 17, déc. 1970), p. 39.

11 Alfred Glauser fait ingénieusement remarquer que, tout en étant voué au culte de la parole, Rabelais a aussi le goût du silence: "Il l'a souvent suggéré par les gestes qui remplacent les paroles." In Rabelais Créateur (Paris: Nizet, 1966), p. 282.

12 A propos des gesticulations de Panurge et de Nazdecabre, il est important de lire: François Rigolot, Les languages de Rabelais, Etudes rabelaisiennes (Droz, 1972), en particulier p. 49.

13 Saussure définit la sémiologie comme la science "qui étudie la vie des signes au sein de la vie sociale." In cours de linguistique générale (Paris, Payot, 1972), p. 33. Il faut noter que Saussure n'emploie jamais le mot "signal."

14 Eric Buyssens, La communication ct l'articulation linguistique (Presses Universitaires de Bruxelles, 1967), p. 64.

15 Emile Benveniste, Problèmes de linguistique générale (Gallimard, 1966), p. 27. 